\title{
5GinFIRE: An End-to-End Open5G Vertical Network Function Ecosystem
}

\author{
Aloizio P. Silva ${ }^{\mathrm{d}, 1, *}$, Christos Tranoris ${ }^{\mathrm{b}}$, Spyros Denazis ${ }^{\mathrm{b}}$, Miguel Luís ${ }^{\mathrm{c}}$, Susana \\ Sargento $^{\mathrm{c}}$, João Pereira ${ }^{\mathrm{c}}$, Rodrigo Moreira ${ }^{\mathrm{d}}$, Flávio Silva ${ }^{\mathrm{d}}$, Ivan Vidal ${ }^{\mathrm{e}}$, Borja \\ Nogales $^{\mathrm{e}}$, Reza Nejabati ${ }^{\mathrm{a}}$, Dimitra Simeonidou ${ }^{\mathrm{a}}$ \\ ${ }^{a}$ University of Bristol, Bristol, United Kingdom \\ ${ }^{b}$ University of Patras, Patras, Greece \\ ${ }^{c}$ Instituto de Telecomunicações - Aveiro, Portugal \\ ${ }^{d}$ Federal University of Uberlândia (UFU), Uberlândia, Brazil \\ ${ }^{e}$ University Carlos III of Madrid, Madrid, Spain
}

\begin{abstract}
Advanced communication networks, such as $5 \mathrm{G}$ and beyond, will be a complex ecosystem made of multiple physically interconnected elements, implying that the upcoming network will have to address capabilities such as flexibility, programmability and extensibility. This article, describes an Open and Extensible 5G Network Function Virtualisation (NFV) based Reference ecosystem of experimental facilities, named 5GinFIRE, that integrates existing facilities with new vertical-specific ones but also lays down the foundations for instantiation fully softwarised architectures of vertical industries and experimenting with them. Additionally, we present 5GinFIRE as the forerunner experimental playground, together with three uses cases, wherein new components, architecture designs and APIs may be tried and proposed before they are ported to more industrially mainstream $5 \mathrm{G}$ networks that are expected to emerge in large scale.
\end{abstract}

Keywords: Network Function Virtualization, 5G, Experimentation Platform, Network

\footnotetext{
* Corresponding author

Email addresses: aloizio@northeastern.edu/ aloizio.dasilva@us-ignite.org (Aloizio P. Silva), tranoris@ece.upatras.gr (Christos Tranoris), sdena@ece.upatras.gr (Spyros Denazis), nmal@av.it.pt (Miguel Luís), susana@ua.pt (Susana Sargento), pt@av.it.pt (João Pereira), rodrigo.moreira@ufu.br (Rodrigo Moreira), flavio@ufu.br (Flávio Silva), ividal@it.uc3m.es (Ivan Vidal), bdorado@pa.uc3m.es (Borja Nogales), reza.nejabati@bristol.ac.uk (Reza Nejabati), dimitra.simeonidou@bristol.ac.uk (Dimitra Simeonidou)

${ }^{1}$ Northeastern University, Boston, Massachusetts, US, Since 2019.
} 
Softwarization, Vertical Industry

2019 MSC: 00-01, 99-00

\section{Introduction}

Key industrial sectors, such as manufacturing, automotive, energy, entertainment industry to name a few, are rapidly being transformed by digital and communication technologies leading to the fourth industrial revolution. New ones are in the making, i.e., smart cities, which inspire a new breed of applications and services. The salient characteristic of these verticals is that they do not operate and evolve in a silo mode anymore, relying on closed and proprietary technological environments, but they are becoming open ecosystem built on top of shared physical infrastructures, (re)using the same open source components and APIs and sharing resources utilizing virtualization. This context presents a number of multi-facet challenges (such as interoperability, inter-connectivity, federation, resource sharing, slicing, resource scheduling, operations) of unprecedented magnitude and complexity and it calls for the appropriate identification, introduction, integration and efficient operation of a series of common architectural elements and infrastructure assets that should be combined together with the vertical-specific components. It also calls for an environment engineered in such way that is capable of co-hosting different verticals while addressing competing requirements of applications and services deployed and run in the context of the various verticals. In this direction, this article presents 5GinFIRE ecosystem which is motivated by the previous described context and two interlinked questions.

1. How such a holistic and unified environment should look like?

2. How can 5GinFIRE host and integrate verticals and concurrently deal with reconciling their competing and opposing requirements?

Addressing these key questions, 5GinFIRE main technical objective is to deploy and to operate an open, and extensible 5G NFV-based reference ecosystem ${ }^{2}$ of experimental facilities that not only instantiates facilities with new vertical-specific ones

${ }^{2}$ 5GinFIRE Portal: https://5ginfire.eu/ 
but also lays down the foundations for instantiating fully softwarised architectures of vertical industries and experimenting with them. The instantiation of the 5GinFIRE ecosystem is as generic as possible in order to host any kind of verticals.

In order to guarantee architectural and technological convergence the 5GinFIRE environment has been built in alignment with on-going standardization (i.e. European Telecommunications Standards Institute (ETSI) NFV) and open sources (i.e. OPEN MANO) activities, also targeted by other closely related programme activities such as 5G-PPP ${ }^{3}$ In particular, 5GinFIRE ecosystem serves as the forerunner experimental playground wherein new components, architecture designs and APIs may be tried and proposed before they are ported to more industrially mainstream $5 \mathrm{G}$ networks that are expected to emerge in large scale. So far as we know, 5GinFIRE is the first platform that strongly and uniquely places key standardization activities in the core of its concept. Contributing and enhancing the standardization activities in 5G NFV and offering a unique standardized way of setting up and running experiment for advanced network communities across 5GinFIRE facilities.

In the next section, the state-of-the-art of 5G technologies and platforms are briefly reviewed, following by the 5GinFIRE ecosystem description such as: target areas, 5GinFIRE architecture and overall methodology, blueprint uses cases and final remarks.

\section{2. Related Work}

The digital transformation of network infrastructure through NFV and software defined networking (SDN) promises to play a key role with the respect to the commercialization of $5 \mathrm{G}$ and the digitalization of vertical markets. There are several major architectural issues facing 5G networks, which can only be overcome leveraging NFV and SDN.

The basic idea behind NFV and SDN is to decouple software from hardware enabling flexibility, programmability and extensibility of the network. With NFV, service

\footnotetext{
$\sqrt[3]{\text { https: }: / / 5 g-p p p . e u /}$
} 
providers can deploy various Network Functions (NFs), such as firewall or encryption, on virtual machines (VMs). Whenever a customer requests a new NF, service providers are able to spin up a VM for that function automatically. Leveraging this technology, network administrators do not need to invest in high-priced, proprietary hardware to set up a service chain of network-connected devices. And unlike proprietary hardware, these NFs can be installed almost instantaneously.

Many standardization efforts are currently on-going, the most prominent being the European Telecommunications Standards Institute (ETSI) ${ }^{4}$ NFV. This standardization activity follows a dual approach, which benefits on the one hand from top-down design documents and requirements and on the other hand Proof-of-Concept $(\mathrm{PoC})$ demonstrators and prototypes that showcase the aspects under standardization as well as the feasibility of the approach. PoCs usually team together vendors with complementary expertise (i.e. a network vendor and a software house specializing in virtualization or cloud technologies). However, the PoCs are meant for illustrative purpose only, and are typically implemented in a closed environment, which is vendor-specific. This does not allow PoCs to be comparable and they become one-of-a-kind showcase, as opposed to a foundation for further development. With the introduction of components developed in the context of vertical industries, the PoC approach is not sufficient anymore and it needs to be expanded to account for more complex vertical-specific service architecture that must coexist and share the underlying infrastructure substrate and resources therein. This is also stressed in the 5G-PPP white paper [5] and the 5 layered integrated 5G architecture for mobile broadband and vertical services. 5GinFIRE offers an open environment for verticals by establishing an industry-led and industry-focused distributed and multi-domain NFV infrastructure fabric. An environment implemented following the standardized NFV ETSI reference architecture based on Open APIs and Open Source services, which complements the planned activities in ETSI and 5G-PPP for vertical services development and demonstration. In addition, it provides a forerunner experimental platform for FIRE + engagement in ETSI standardization, 5G-PPP, and future market transfer.

\footnotetext{
$\sqrt[4]{\text { http: //www.etsi.org/ }}$
} 
Previous network and Future Internet Research \& Experimentation (FIRE) 5 projects have created a lot of know-how in Europe and deployed novel software and hardware facilities, but have had limited adoption by industry leaders, let alone the creation of a single source of innovation in the European SDN and NFV environments. Further, FIRE projects by and large have been distant to standardization activities, let alone used for demonstrating new possibilities with emerging standards. Instead, we have seen several open source projects emerging from large corporations that bypass FIRE facilities.

Although NFV is meant to lower the entry barriers to smaller players, practically, the current PoC effort is dominated by large players with limited to almost no Small and Medium-sized Enterprise (SME) involvement. The situation is further aggravated when dealing with innovation targeting specific verticals as the experimentation environment is highly isolated from each other as well as specialized, while offering a few and fixed services for experimentation. Exploiting the potential of softwarization of almost every aspect of functionality, network or application specific, we can foster SME innovation on top of 5GinFIRE ecosystem for experimentation and rapid prototyping in the area of network service virtualization for verticals and application thereof.

Currently NFV vendors or/and users independently implement numerous NFV platforms and solutions. This results in the dilution of efforts and fragmentation of technologies to be produced leading to divergence and gaps impacting interoperability, and, ultimately, the openness of platforms. To this end, 5GinFIRE acts as the playground for technology convergence enabling more permanent collaboration link with initiatives such as ETSI, OPENFV 6 , FIRE, 5G-PPP and FIWARE.

The 5G Telefonica Open innovation Laboratory (5TONIC) [2] is used by 5GinFIRE platform to offer access to specific-purpose hardware, to assist in experiments, trials and demonstrations with $5 \mathrm{G}$ network technologies, as well as to commodity hardware which allows a cost-effective approach to configure different network topologies of variable size and capacity.

\footnotetext{
5 https://www.ict-fire.eu/projects/

(https://www.docker.com/
} 
FIWARE 7 platform provides a rather simple powerful set of APIs that ease the development of smart applications in multiple vertical sectors. The specification of these APIs are public and royalty-free. However, FIWARE is far from an open NFVbased reference platform. FIWARE focuses primarily on providing high level APIs targeting at specific applications through a concept called domain specific enablers.

A number of projects (SEMAFOUR [10], NOVI [7], 4WARD [1], SAIL [9], TNOVA [12], UNIFY MCN [13], ALIEN [3]) fall within scope of SDN and NFV. Although they address specific aspects of the 5GinFIRE architecture framework they are rather predecessors of 5G-PPP projects or they had a specific focus. Under any circumstances, these and the 5G-PPP projects may use the 5GinFIRE experimentation environment as a template that can host their outcomes. This will promote reusability of project results facilitated by FIRE solution.

[18] provides a brief overview of NFV, explains its requirements and architectural framework, presents several use cases (i.e. virtualisation of mobile core network, home network), and discusses the challenges (i.e. network performance of VNF, VNF placement and migration, VNF outsourcing) and future directions. The authors pointed out that it is envisioned that NFV, along with cloud computing and SDN, will become a critical enabling technology to radically revolutionize the way network operators architect and monetize their infrastructure. 5GinFIRE follows this vision.

The Fraunhofer FOKUS OpenSDNCore [14] is a platform similar to 5GinFIRE that acts as an end-to-end middleware between:

- A distributed heterogeneous infrastructure including dedicated components (e.g. radio), heterogeneous data centers (compute storage) and inter-connecting networks (fronthaul, backhaul, third party backbone, etc.)

- Generic network functions implemented in software and running in virtual machines: Virtualised IMS, EPC, radio and SGi components, home and enterprise networks, Application Servers, etc.

OpenSDNCore is a practical implementation of the future network evolution paradigms,

/https://www.fiware.org/ 
realizing Network Functions Virtualisation (NFV) and Software Defined Network (SDN) concepts matching with the 5GinFIRE principles. It also is based on ETSI NFV MANO aligned Orchestrator integrated with OpenStack enabling the dynamic deployment and run-time management of virtual network functions.

In [19] is presented an architecture of an SDN/NFV network and cloud computing platform for end-to-end 5G services. This platform integrates ADRENALINE, GEDOMIS and EXTREME testbeds, three complementary testbeds developed by CTTC, spanning from terminals to radio access network, aggregation/core networks and cloud. 5GinFIRE is also based on multi-domain orchestration offering dynamic and flexible end-to-end connectivity and virtual network provisioning services across multi-domain and multi-technology networks.

The trend for $5 \mathrm{G}$ future networks is clearly dominated by softwarization at all levels, starting from Software Defined Radio (SDR) including Dynamic Spectrum Sharing (DSS), SDN, NFV up to a software defined holistic environment for technical and business innovation integrating networking, computing and storage resources into one programmable and unified infrastructure. 5GinFIRE has similarities and is aligned with SOFTFIRE [11] and ORCA [8] projects regarding to the trends for 5G experimentation. SofFIRE looks for bringing NFV and SDN capabilities in order to create a reliable, secure, interoperable and programmable experimental network infrastructure. Such environments are used to assess the maturity and industrial viability of these technologies by evaluating system properties in terms of efficiency, functional responsiveness (expressed in terms of measurable Key Performance Indicators (KPIs)) and the ability to create new applications on the platform. ORCA [8] is an end-to-end network experimentation that focus on open and modular software and hardware architectures available that smartly use novel versatile radio technology, more-specifically real-time SDR platforms meeting the requirements in terms of runtime latencies, throughput, and fast reconfiguration and reprogramming. In order to meet at the same time diverging Quality of Service (QoS) requirements over wireless networks, control mechanisms are introduced by ORCA platform that allows the configuration and deployment of optimized radio slices that can be mapped to virtual network slices configured by SDN, as such realizing a joint SDR-SDN paradigm. On the other hand, 5GinFIRE introduces 
business innovation over such transforming communication technologies leading to so-

lution for verticals that are rapidly forming open ecosystems built on top of open common infrastructures and resources. This requires a high degree of technological convergence among vertical industries empowering them with enhanced technical capacity to trigger the development of new, innovative products, applications and services. In order to guarantee architectural and technological convergence the experimentation environment must be in alignment with on-going standardization and open source activities inherently forming a forerunner experimental playground for emerging "mainstream" 5G networks which are defined through 5GinFIRE's goal.

\section{5GinFIRE Architecture}

5GinFIRE ecosystem uniquely offers virtualized elements for building complex constellations of virtual functions, all running on a mix of real and virtual network or computing elements. However the biggest challenge in realizing such an open network substrate is "interoperability" which needs to be explicitly addressed at the architecture specification phases.

Interoperability, in terms of orchestration, deployment and Virtual Functions operations, can be achieved through standards specifications, which are necessary to be complemented with actual implementations (PoCs) in the form of open operational reference platforms that validate the standards and act as a showcase or experimentation environment for applications and services for feedback generation.

ETSI's NFV reference architecture and PoCs [17] are an initial attempt to address the issues above while Open NFV open source project (OPNFV) will provide reference software implementations of architectural components of the former. 5GinFIRE overall concept is based on ETSI's NFV reference architecture but in the same time it semantically enriches and extends it in many ways to account for vertical specific requirements and functionalities. As an example, resource scheduling, experimentation planning, experiment operations, VNF versioning, VNF/NSD validation are aspects that are not addressed by the ETSI NFV architecture. It also bridges the gap between verticals operation and execution of experiments since by following standard- 
ized deployment mechanisms the same experimentation VNFs can be re-deployed in an operational environment as is. As a result, Figure 1 shows the 5GinFIRE reference model architecture which is composed of four building blocks. These blocks supports the life-cycles of verticals (deployment, instantiation, execution, control and release).

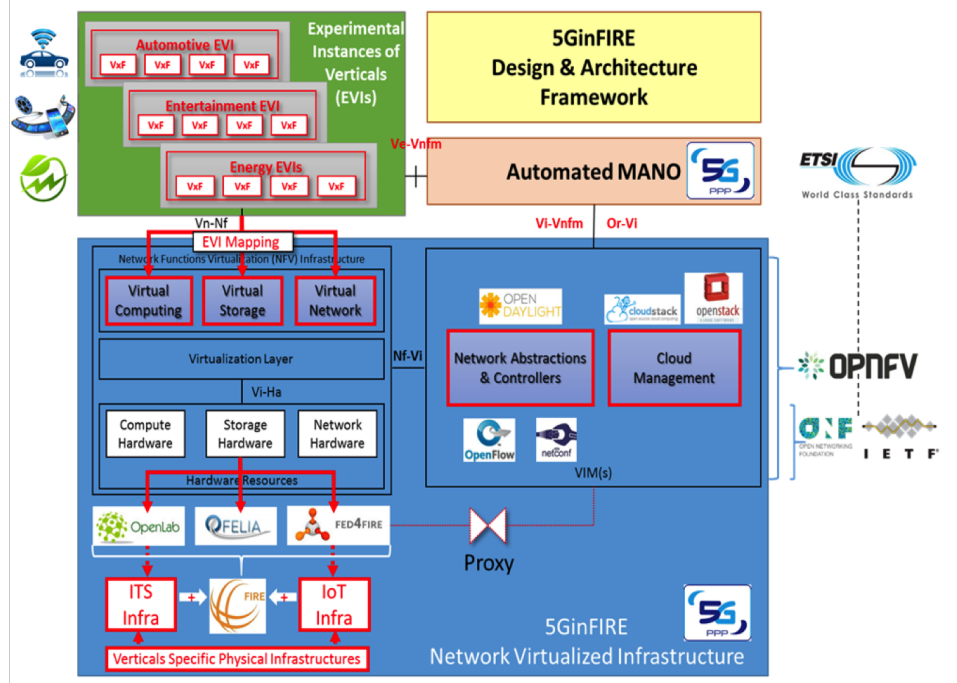

Figure 1: 5GinFIRE reference architecture.

1. Experimental Instances of Verticals (EVIs): these EVIs include a composition of several virtual functions (network or vertical) spanning all layers from application and services to networking. We refer to these virtual functions as $V x F s$ without distinguishing between network-centric functions and vertical-centric functions.

2. 5GinFIRE Network Virtualized Infrastructure (5GNVI): 5GNVI is comprised of facilities that offer raw resources, namely, computational, network and storage which are shared among several VxFs. These resources are offered as virtual/physical resources by the underlying hardware through a virtualization layer that extends itself across a combination of mainstream physical infrastructures such as Cloud Computing and vertical specific ones e.g. Intelligent Transportation Systems (ITS) including the vehicles themselves that host Mobile Edge Computing functionality. Management of the operations of the infrastruc- 
tures and of the corresponding resources is carried out through the Virtualised Infrastructure Managers (VIMs) while control. As 5GNVI may span across several locations, i.e. places operated by different testbed providers, multiple VIMs may exist, one per testbed site. Although the scope of VIMs is local they are accessible through well- established APIs and their implementation relies on open source which facilitate their integration and intercommunication for any distributed EVI functionality.

3. Automated Management and Orchestration (auto-MANO): auto-MANO building block focuses on the orchestration and life-cycle management of the 5GNVI in a global manner.

4. 5GinFIRE Design and Architecture Framework: includes the APIs for creating and experimenting on new services and applications facilitating the integration with the industrially led standardization and open source activities.

5GinFIRE architecture is composed of the core network and heterogeneous NFVIs, also called facilities, that spread over different locations. Both constitute the overall 5GinFIRE ecosystem that can be integrated with existing testbeds such as FIRE facilities.

Provisioning and running of network services and applications in FIRE in the form of experiments are important operations offered by various FIRE facilities, past and present. This gives rise to an overlapping area with industrially led initiatives such as OPENFV and 5G- PPP that needs to be addressed properly in order to bring mutual benefits. As the need for creating, experimenting on new services and applications is ever increasing and is expected to further intensify with the addition for vertical support, it calls for a radically different underlying physical network infrastructure that extend horizontally like in the case of $5 \mathrm{G}$. Such requirement is hindered by the availability of specialized hardware and/or proprietary network software which raises serious open API accessibility and configuration issues in hard-to-find testbed infrastructures. Although, federation approaches, as in Fed4FIRE, in particular, in accessing and using FIRE facilities, may mitigate the problem, they are far from solving it as they confine themselves to managing stove- piped experimental facilities. 5GinFIRE 
is aware of this issue and dedicates special attention on how existing FIRE know-how and facilities could be seamlessly integrated with the industrially led standardization and open source activities. This is the purpose of the architecture component, called Proxy (see Figure [? ]), which aims at facilitating technological convergence of FIRE with future $5 \mathrm{G}$ operational platforms.

\subsection{Core architectural components}

5GinFIRE offered services and tools target to accommodate the following envisaged user roles. All users are assumed to be of an Authenticated role:

- Experimenter: This role represents the user that will utilize 5GinFIRE services and tools to deploy an experiment.

- VxF Developer: This role is responsible to upload VNF and NSD Descriptors in the 5GinFIRE services.

- Testbed Provider: This role represents users that are responsible for testbed administration, configuration, integration, adaptation, support, etc.

- Experiment Mentor: responsible for monitoring the progress of an experiment, resource usage and allowing or not the deployment of an experiment.

- Services administrator: This role represents the user that are responsible for maintenance of the 5GinFIRE services.

Figure 2 shows the core architectural components and their interfaces for experimentation in 5GinFIRE platform.

- Portal: 5GinFIRE portal is a web application where end-users (Experimenters, $V x F$ Developers) can subscribe, manage experiments, browse repository, monitor experiment results, etc. In addition, it allows admins to manage the 5GinFIRE platform and the VNF/NSD repositories. In particular, the 5GinFIRE portal provides access to the 5GinFIRE repositories of $V x F s$ metadata and descriptors (e.g. Unifier Gateway VNFs), categorized in EVIs (e.g. Networking, Automotive, Media) through well specified APIs. Three interfaces exist in the portal: 


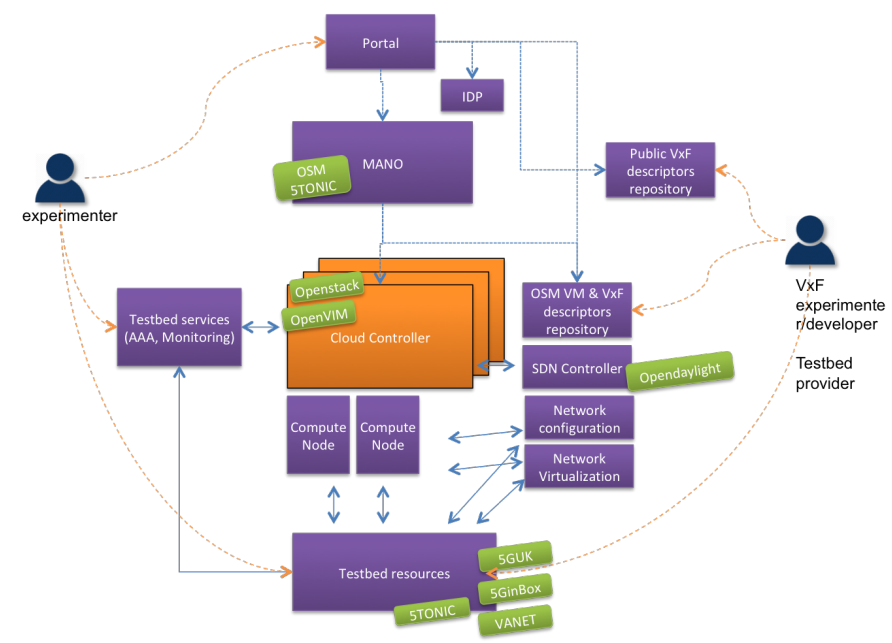

Figure 2: 5GinFIRE high level experimental architecture.

1. OSM: this interface allows to communicate with OSM MANO in order to push experiment description and $V x F$ descriptors.

2. IDP: is an identity provider mechanism for authenticating 5GinFIRE users. IDP has two interfaces: 1) Portal for authenticating portal users and; 2) Support tools for ticketing system authentication.

3. Public $V \boldsymbol{x} F$ descriptor repository: contains all $V x F s$ registered by 5GinFIRE users. It has one interface with the portal that will accept request of managing $V x F$ descriptors and their archives.

4. OSM VIM \& $\boldsymbol{V} \boldsymbol{x} \boldsymbol{F}$ descriptor repository: is the OSM repository where $V x F$ that will be instantiated need to be committed.

- 5GinFIRE MANO platform: MANO platform, which is based on OSM as section B describes, receives orchestration actions from 5GinFIRE Portal (i.e. to create/delete a VNFD in/from the OSM catalog, to create/delete an NSD in/from the OSM catalog, to instantiate a NS, etc). The 5GinFIRE MANO platform has interfaces to the portal and towards the VIM endpoints, to request the allocation and release of computing, storage and network resources at the partners' NFV Infrastructure.

- VIMs (Cloud Controllers): each partner providing an experimental infrastruc- 
ture to 5GinFIRE is in charge of the deployment and maintenance of a VIM supported by the 5GinFIRE MANO platform. Each VIM deployed at a partner infrastructure domain must provide a compliant northbound API to 5GinFIRE MANO platform. This component contains two interfaces:

1. 5GinFIRE MANO: enables the interactions with 5GinFIRE MANO platform.

2. Testbed resources: enables to control and manage the computing, storage and network resources of a NFVI.

- Testbed services: testbed specific services that can be handover to the experimenter in order to facilitate the operations during the experimentation.

- Testbed resources: the available resources for experimentation located in each target testbed. For instance:

- 5GUK Test Network [4] is a multi-site solution connected through a citywide single mode fibre ring with several active switching nodes. The core network is located at the High-Performance Network (HPN) research group laboratory at the University of Bristol with access technologies located in Millennium Square for outdoor coverage and We The Curious science museum for indoor coverage.

- 5TONIC includes a solid baseline of facilities and infrastructure that supports advanced experimentation in the area of $5 \mathrm{G}$ network technologies. It offers a data center with multiple communication racks, a VPN access service and high-speed network connectivity with external networks. Through RedIRIS [15] and GÉANT [6] Communication racks are allocated to individual 5TONIC members, and may be flexibly interconnected according to any experimentation requirements. Additionally, 5TONIC provides its members with access to a common infrastructure with specific-purpose hardware, to assist in experiments, trials and demonstrations with 5G products and services. 
- 5GinBox: 5G-In-a-Box, also called Unifier Gateway is a set of VNFs providing various radio access technologies and core network features by mechanism to enable new sites (a.k.a. testbed providers) interconnections to the 5Gin- 
FIRE ecosystem. OSM is responsible to deploy the $V x F s$ accordingly defined to each experiment. This approach allows for the evolution of the ecosystem to support the three main horizontal lines: the experimenter, the 5GinFIRE operations and the 5GinFIRE testbed providers that interact during an experimentation life-cycle. In order to instantiate experimentation scenarios end-users/experimenters must use 5GinFIRE

Figure 2 details connectivity between 5GinFIRE testbed providers and the intersite data exchange between these facilities. The network addressing plan is based on pre-defined separated subnets within the same broadcast domain. Each site has a L3 entity that provides an overlay network connection to the VPN server. The yellow line is an enabling channel to exchange control information among OSM, SDN and remote VIMs. The exchanged messages in this channel allow deploy of the required VxFs. Additionally, the green line enables OSM to communicate directly with the deployed $V x F$ to execute the necessary settings. Also, the purple line is the channel of the data plane, to allow communication between VxFs. Note that the data plane distribution model is hub-and-spoke, star topology centered on the 5TONIC. It is possible for sites to establish bilateral data plane connectivity, without fundamentally being centralized, this autonomy supports specific requirements of $5 \mathrm{G}$ verticals.

To validate the connectivity, a set of functional tests are pre-defined to integrate a new testbed provider in the 5GinFIRE ecosystem. These functional and experimental tests are done in threefold stages: i) validate the capacity of the overlay network, ii) ability to launch VNFs on remote site and iii) testing the NS deployment.

\section{5GinFIRE Methodology}

The reference architecture presented in the previous section supports the methodology that guides the experimentation on top of the 5GinFIRE platform.

Figure 3 displays an overview of the experimentation workflow process. There are Portal, thus users sign-up to the platform via the portal. The experimenter needs to compose the experimentation description through an ETSI compliant Network Ser- 


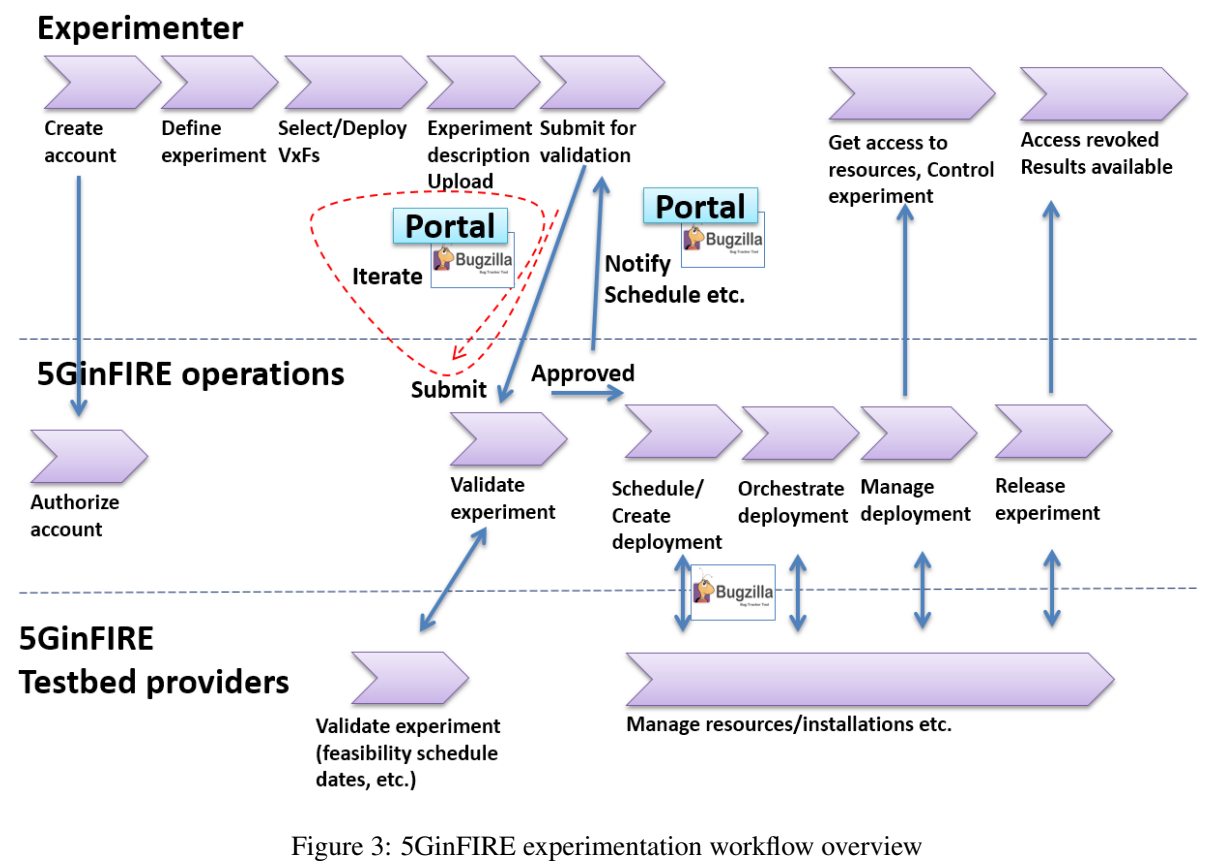

vice Descriptor (NSD) composed of VNF Descriptors (VNFD) based on YAML 8 that the OSM will accept in order to instantiate the experimentation scenario and submit it to the portal. Then NSD is validated for archive compliance and deploy-ability and then is on-boarded to OSM. As soon as everything is in place and valid for an experiment deployment, the experimenter selects the testbed facility (or facilities for inter-connectivity experiments), places the VNFs to target facilities, defines experiment metadata, scheduling, purpose and submits it for approval.

5GinFIRE operations team has a process for approving an experiment in terms of various rules such as schedule, resource availability, etc. The approval process is closely performed together with the target testbed providers. To properly handle the process the 5GinFIRE operations assigns a so called Experiment Mentor that will approve and monitor the experiment.The Mentor drives discussions between experimenters and VIM owners and also verifies that the requested experiment is deployable. This implies, at least: checking that all the components are onboarded; and checking

\footnotetext{
${ }^{8}$ http://yaml.org/
} 
that the VNFs can be executed in the involved testbeds (e.g., a VNF for an EPC should be executed in a testbed with radio equipment).5GinFIRE has already setup an automated validation process via Continuous Integration techniques but there are cases that the request is not feasible (e.g. placement on Edge/Gateway devices). The Experiment Mentor also coordinates and provides a specific time slot for the execution of the experiment. This time slot may be different from the time slot requested by the experimenter depending on resources availability. All stages are traced from our ticketing system based on Bugzilla, which informs the operations team and all relevant stakeholders (experimenter, testbed providers, etc). As soon as an experiment is approved by the Experiment Mentor, the 5GinFIRE operations create a deployment request, onboards the NSD to OSM and OSM will orchestrate it. In all stages there is a close collaboration during the management of the orchestration/deployment with the testbed providers. After NSD instantiation the resources are available and accessible to the experimenter. In the end of the experiment schedule, the resources of the experiment are released and access is revoked. Any available results of the experiment might be available to the experimenter (e.g. infrastructure monitoring) when necessary.

\section{Experimentation Enablement}

In order to make sure that all the necessary functionality and corresponding facilities have been properly developed, integrated and operating and they support verticals experimentation, some internal pilot uses cases have been designed and implemented to validate the $5 \mathrm{GinFIRE}$ ecosystem. The outcomes of this internal uses cases have produced some templates and $V x F s$ for verticals that provide the basis for future experiments. The next sections briefly describe each one of these uses cases.

\subsection{Car Overtaking}

This section describes the car overtaking use case that is part of the automotive EVI. The use case's objective is to gather real-time information to assist the driver in critical situations, e.g. car overtaking scenarios with reduced road visibility. To help the read driver in these situations a live stream from the front vehicle is transmitted to the rear 
vehicle. However, to cope with the possibility of the targeting vehicle not behind able to support the original video format, a video transcoding operation must occurs. Thus, a video transcoder $\mathrm{VxF}$ was developed and deployed at the edge via 5GinFIRE portal.

The architecture of the automotive car overtaking use case is illustrated in Figure 4. Each vehicle contains a video camera on its front side and an On-Board Unit (OBU) capable of providing two different communication technologies: IEEE $802.11 \mathrm{p}$, enabling the communication between vehicles $(\mathrm{V} 2 \mathrm{~V})$, and between vehicles and the infrastructure (V2I), and 4G/5G cellular as a complement technology to be used in the absence of IEEE 802.11p communication links. The OBU is connected with an In-Car Node Processor that will be used to process and provide visual information to the driver. On the infrastructure side, each Road Side Unit (RSU) is connected to the automotive testbed datacenter, located in Aveiro, Portugal, where the VNFs are located. The 4G/5G cellular network is operating under the concept of Cloud-RAN (C-RAN), and supported by a VNF cellular unified gateway also deployed at the automotive testbed datacenter. However, for the results here presented only IEEE 802.11p communication links were used.

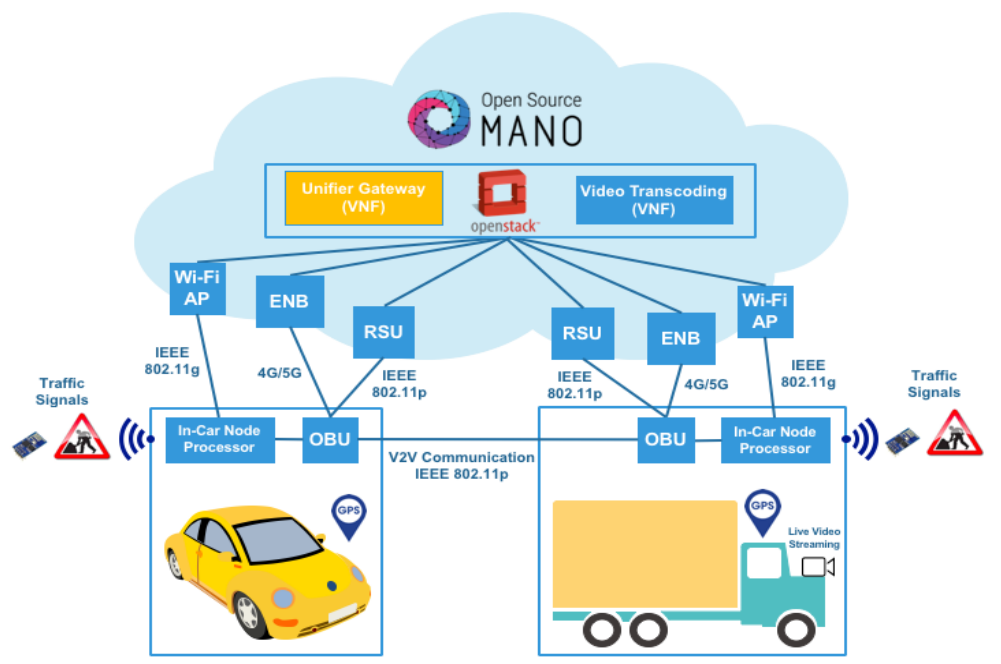

Figure 4: Car overtaking experimentation architecture. 
To assess the functionality and performance of the video transcoding $\mathrm{VxF}$, two distinct scenarios were explored. However, in both scenarios the connectivity map is the same consisting of two RSUs and two OBUs: the rear OBU is connected to a rear $\mathrm{RSU}$ and the front $\mathrm{OBU}$ is connected to a front RSU, i.e. no direct connectivity exists between both OBUs. For experimentation puroposes, RSUs are connected through Ethernet cable. In the first scenario it is assumed that no video transcoding is required and once the video feed reaches the front RSU it is streamed directly to the rear RSU, and then to the rear vehicle. Once the rear OBU receives the video stream it sends the video to the visual screen in the car, giving the driver the possibility to have realtime access to visual information. In a second scenario the functionalities of the video $\mathrm{VxF}$ transcoder are explored. For that, once the video stream reaches the front RSU it goes to the video VxF transcoding located at IT-Aveiro's datacenter. From there, the transcoded live feed is sent to the rear RSU and then to the rear vehicle, using IEEE 802.11p.

Table 1 presents the average latency and Received Signal Strength Indicator (RSSI) observed during the experiments. The latency was measured between OBUs while RSSI values were measured on each IEEE $802.11 \mathrm{p}$ link, i.e. between the front OBU and the first RSU, and between the rear OBU and the second RSU. As expected, the results show that the lowest latency is observed in the shortest communication path. In fact, with the introduction of the $\mathrm{VxF}$ video transcoding, performed in the cloud, the latency increased 50 times due to the time needed to perform this operation (the average transcoding delay was $980 \mathrm{~ms}$ ). As for the RSSI, the results are very similar on each experiment. The RSSI between the rear vehicle and its RSU is higher than the RSSI between the front vehicle and its RSU mainly due to the distances observed during the experiments.

The results presented in Table 1 were obtained in an urban scenario where the average velocity is considerably smaller when compared to a highway scenario. In order to understand the impact of vehicle's speed on the link quality, Figure 5 presents the latency observed between each $802.11 \mathrm{p}$ communication, i.e. between rear OBU and its RSU, and between front OBU and its RSU. As expected, higher speeds induce higher delays in the communication, but similar results are observed for when the transmitter 
Table 1: Average latency and RSSI observed during the car overtaking experiment.

\begin{tabular}{c||c||c|c}
\multicolumn{1}{c||}{} & \multicolumn{1}{c||}{$\begin{array}{c}\text { RSSI } \\
\text { end-to-end }\end{array}$} & $\begin{array}{c}\text { Rant OBU } \\
\text { to front RSU }\end{array}$ & $\begin{array}{c}\text { rear OBU } \\
\text { to rear RSU }\end{array}$ \\
\hline \hline $\begin{array}{c}\text { Without VxF } \\
\text { Video Transcoding }\end{array}$ & $19 \mathrm{~ms}$ & 38 & 80 \\
\hline $\begin{array}{c}\text { With VxF } \\
\text { Video Transcoding }\end{array}$ & $1022 \mathrm{~ms}$ & 39 & 79
\end{tabular}

is the OBU and the receiver is the RSU (front OBU front RSU path) and when the transmitter is the RSU and the receiver is the OBU (rear RSU rear OBU path).

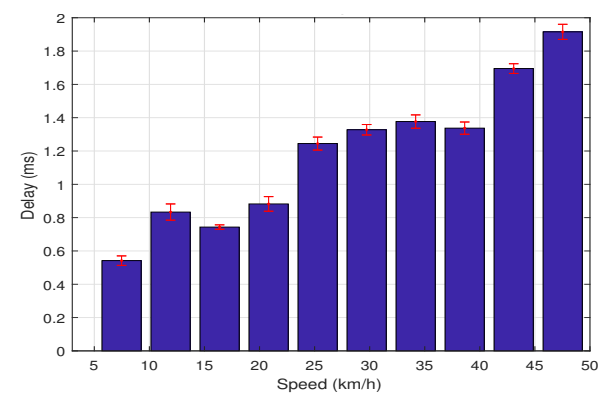

(a) Between rear OBU and rear RSU.

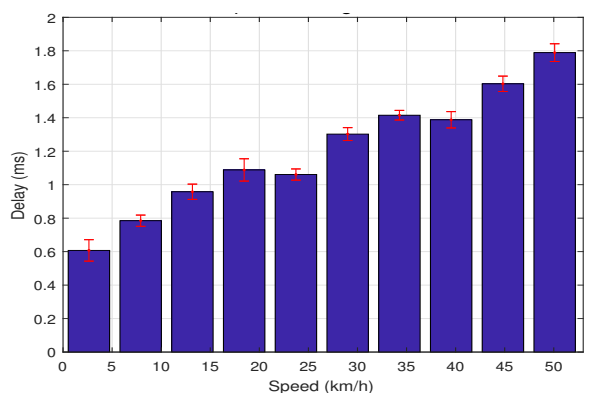

(b) Between front OBU and front RSU.

Figure 5: The impact of the vehicle's speed in the latency of an IEEE 802.11p transmission link.

\subsection{Smart City Safety}

This section describes the smart city EVI where a blueprint smart city use case named Smart City Safety has been demonstrated on top of the 5GinFIRE ecosystem. This use case help to identify criminals around the city by capturing $360^{\circ}$ live stream video and providing face detection and recognition. To this end a VNF video transcoder (based on OpenCV including face detection and recognition program) has been deployed at the datacenter located in Bristol via 5GinFIRE portal. The NSD and VNFD were uploaded in 5GinFIRE Portal that communicates with OSM MANO to deploy the network service at University of Bristol (UNIVBRIS) NFVI. The VNF video transcoder is responsible for processing the live stream video from the spherical to a rectangular format to enable face detection/recognition. 
The VNF video transcoder includes three phases:

1. video trasconder: where the $360^{\circ}$ live stream video is converted to a rectangular format.

2. face detection: it has the objective of finding the faces in an video frame and extract them to be used by the face recognition algorithm. Haar feature-based cascade classifiers is used as an effective object detection method proposed by [23].

3. face recognition: with the facial images already extracted, the face recognition algorithm is responsible for finding characteristics which best describe the image. Local Binary Patterns Histograms (LBPH) [16] is used for face recognition. We used a dataset of faces populated with a set of faces from people of Smart Internet $\mathrm{Lab}{ }^{9}$ following data protection and private security for UK.

Figure 6 shows the smart city safety experimentation architecture at UNIVBRIS NFVI. The main building block of this experimentation is composed of a $360^{\circ}$ camera connected via WiFi 2.4Ghz to a Raspberry PI (Raspi) Model B and both are connected to a $28000 \mathrm{~mA}$ battery and attached to a Bike Helmet. The Raspi is connected to the Cloud via WiFi 5GHz. Basically the $360^{\circ}$ camera captures the live stream video and sends to the Raspi that sends it to the datacenter to be processed. The processing takes place through the VNF video transcoder and the face detection and recognition programs. As a result, the processed live stream video is sent to a screen where faces of people are recognized.

The use case was deployed indoor (in the Smart Internet Lab) and outdoor (Millennium Square in Bristol City Center) 10 .

We perform extensive evaluations of the smart city safety use case running on top of 5GinFIRE ecosystem. The evaluation focuses on testing the 5GinFIRE functionalities and capabilities and, at the same time, the application performance regarding to real time requirements. As an example of our evaluation, Figure 7 shows the Total Response

http://www.bristol.ac.uk/engineering/research/smart/

${ }^{10}$ Smart City Safety Demo: https://www.bristol.ac.uk/engineering/research/ smart/5g-demonstrations/smart-city-safety/ 


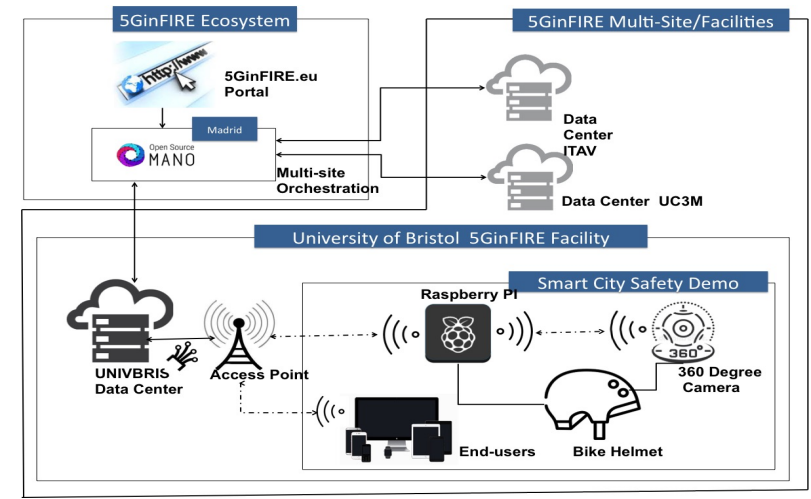

Figure 6: Smart City Safety experimentation architecture.

Time (TRT) for one of the cameras (the results are similar for the other cameras). TRT is the time since the frame is sent from the camera to the datacenter plus the VNF processing time and the time for the video being received at UE. From the real time application point of view, having a small TRT is the main goal. We can observe that the average TRT is $\mu=0.551$ seconds with $\sigma= \pm 0.132$. The TRT values per frame are slightly similar without being affected for high outliers. Resulting in a good QoE.

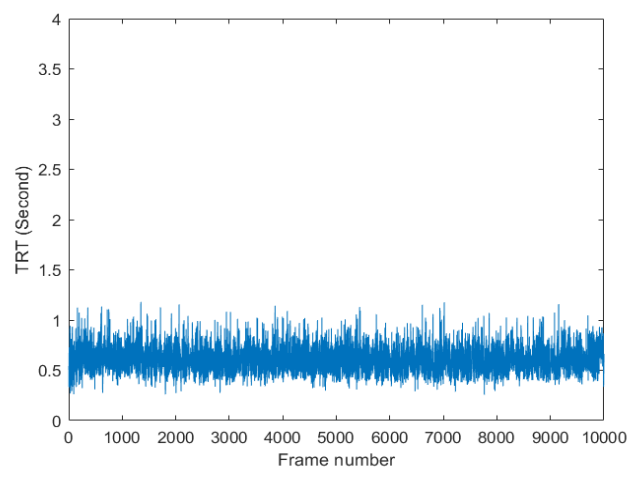

Figure 7: Total response time per video frame.

\subsection{Drones}

Other example of use case developed from the aforementioned platform is an application of the NFV technology to enable the flexible deployments of Unmanned Aircraft Systems (UAS), capable of adapting to different missions in the civil scope. Highlight 
that UAS are composed by a set of Unmanned Aerial Vehicles (UAVs), which are more commonly known as drones. Accordingly, this use case aims at providing the underlying substrate to execute properly networks functions, as well as transport and application functions through virtualization technologies where the computational resources are provided by the UAVs composing the UAS.

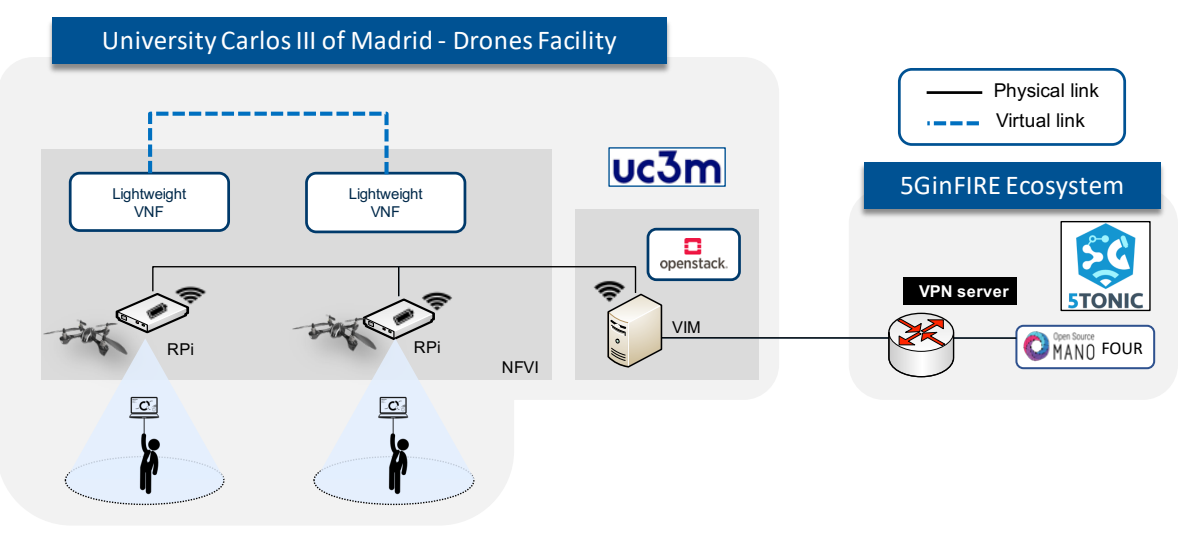

Figure 8: Drones use case architecture

Figure 8 shows the system design employed in this use case, which is mainly composed by three entities; (1) the OSM system, located at 5TONIC and within 5GinFIRE ecosystem, and responsible for orchestrating the deployment of different lightweight VNFs (the term lightweight VNF is referred to those VNFs with low computational cost, capable of being executed in a limited capacity infrastructure as the one offered by an UAV) in the drones facility, as well as the interconnection and configuration of those lightweight VNFs; (2) the hardware and software infrastructure carried out by the UAVs composing the NFVI, which is capable of supporting the execution of the lightweight VNFs; and (3) the VIM in charge of the management of the physical and software resources made available by the NFVI.

This design is provided with a WiFi interface in each UAV, enabling the data exchange with the rest of UAVs that are located within its coverage radio. Additionally, some UAVs can act as access points, offering a common technology to allow the access of users to the services provided by the UAS. Thus, the system design supports 
the execution of lightweight VNFs along with the wireless communications among themselves. In this context, different experiments have been carried out to evaluate the network performance offered in the communications between two lightweight VNFs that are hosted by two different UAVs. The first experiment estimates the end-to-end delay between both lightweight VNFs based on the round-trip time (RTT) measurements collected using the Ping tool. Figure 9 a makes use of the Cumulative Distribution Function (CDF) of the RTT measured and illustrates that the the $90 \%$ of the RTT samples are lower than $11 \mathrm{~ms}$. In a second experiment, the evaluation is focused in the estimation of the bandwidth provided. For this purpose, the throughput is calculated using the Iperf tool and with the CDF of the samples, Figure 9 b shows that the $80 \%$ of the measurements are above $10 \mathrm{Mbps}$ and also that the maximum value is 12.5 Mbps. Comparing the obtained results with [22], where the authors perform an extensive analysis IN the wireless communications of the UAV devices, it can be concluded that the network performance in terms of end-to-end delay and through is not significant affected by the provision of network functionalities through the use lightweight VNFs.
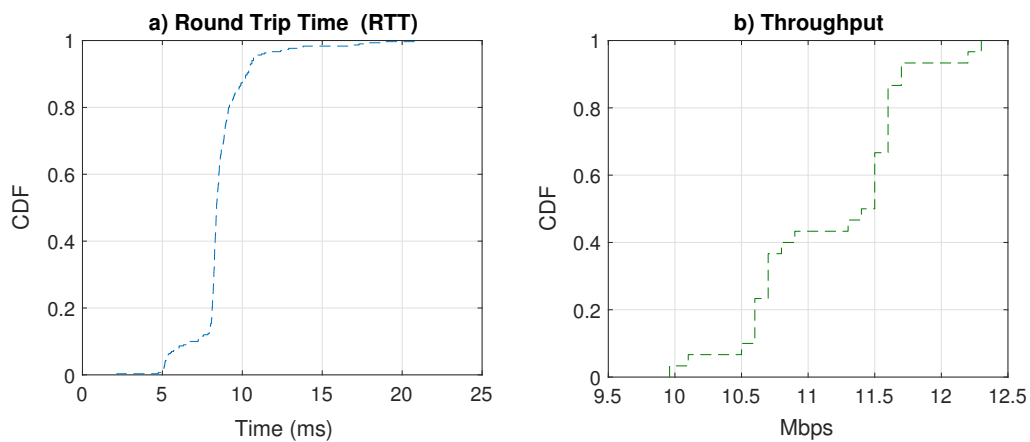

Figure 9: Network performance analysis in the Drones use case

Finally, the implementation of this system design is based on existing open source technologies. Regarding to MANO system, the orchestration solution selected is the one provided by OSM. On the other hand, the cloud computing platform provided by 
OpenStack handles the management of VIM. For the NFVI composed by the UAV, Single Board Computers offered by the Raspi provide the sufficient compute capacity to the UAVs to enable the execution of lightweight VNFs. The lightweights VNFs are implemented using the containers as virtualization technology. This solution offers a more elastic way for implementing function virtualization in the UAS context, since the hardware and software resources provided by the UAVs is limited. The work in [21] and [20] present more in detail the use of UAVs in an NFV environment along with different experiments to validate the feasibility of a system like the already presented throughout this section.

\section{Remarks and Conclusion}

5GinFIRE has established the first 5G NFV-enabled experimental testbed capable of instantiating and supporting vertical industries based on industry-leading and open source technologies. In particular, it creates an open environment for verticals by establishing an industry-led and industry-focused common environment which complements the planned activities in ETSI and 5G-PPP for vertical services development and demonstration and at that same time provides the forerunner experimental platform for FIRE+ engagement in ETSI standardization, 5G-PPP, and future marker transfer.

As we are in the dawn of softwarisation of whole vertical service architectures which are then deployed across NFV-enabled infrastructures, it is important to explicitly deal with specific verticals in order to understand the dynamics behind the lifecycle process of deploying and operating them. It is also equally important to establish best practices of how a vertical service that is usually hosted in closed environments, it should break down into, or composed of components that are distinct and can be combined on demand. 5GinFIRE has specified, implemented and deployed automotive and smart city vertical which can be instantiated on top of the common experimental facilities and customized for targeted experimentation.

The specification of experiments or, equivalently, of the service architecture of verticals followed by the deployment, instantiation, and operation across $5 \mathrm{G} \mathrm{NFV-}$ enabled infrastructure substrate and vertical-specific physical facilities require the effi- 
cient coordination and interoperation of a multitude of functional components known as MANO functionality. MANO covers the orchestration and life-cycle management of experiments, which coincide with the life-cycle management of VNFs from the NFV perspective due to the fact that 5GinFIRE shares the same requirements and architecture framework as ETSI NFV. In this case, 5GinFIRE has integrated MANO open source components and has increased the level of automation in instantiating any virtual function component for experimentation, not just VNFs, including $V x F s$. This has a great impact on innovation as it gives rise to a modular and pluggable system wherein more sophisticated functionality pertaining to MANO architectural components or service verticals can be tested.

In the spectrum from idea to application, 5GinFIRE is a strong contender for the swift development of marketable products, and yet, with a significant degree of innovation stemming from recent research in the field of FIRE technologies and experimentation tools and facilities. We believe that 5GinFIRE is in a advantageous positioning for the development of innovative end-to-end 5G experimentation products, Vertical deployments and integrations that take into account various factors such as practical feasibility, sustainability and market growth.

\section{Acknowledgements}

This article has been supported by the European H2020 5GinFIRE project (grant agreement 732497).

\section{References}

605 [1] Architecture and design for the future internet. http://www. 4ward-project.eu/, 2018. acessed in: May 13rd, 2018.

[2] An open research and innovation laboratory focusing on 5G technologies. https://www.5tonic.org/, 2018. acessed in: May 13rd, 2018.

[3] Abstraction layer for implementationof extensions in programmable networks. http://www.fp7-alien.eu/, 2018. acessed in: May 13rd, 2018. 
口[4] 5GUK test network. http://wWw.bristol.ac.uk/engineering/ research/smart/projects/uk-5g/, 2018. acessed in: May 24rd, 2019.

[45] 5G empowering vertical industries. https://5g-ppp.eu/wp-content/ uploads/2016/02/BROCHURE_5PPP_BAT2_PL.pdf, 2018. acessed in: May 13rd, 2018.

[6] GÉANT Association. https://www.geant.org/, 2018. acessed in: May $23 r d, 2019$.

[1] [7] Networking innovations over virtualized infrastructures. http://www. fp7-novi.eu/about-the-project, 2018. acessed in: May 13rd, 2018.

[8] Orchestration and reconfiguration architecture. https://wwW . orca-project.eu/\#, 2018. acessed in: May 13rd, 2018.

¿ [9] Scalable and adaptive internet solutions. http://www.sail-project. eu/, 2018. acessed in: May 13rd, 2018.

[10] Self-managed system for unified heterogeneous radio access network. http: //wWw. fp7-semafour.eu/, 2018. acessed in: May 13rd, 2018.

[11] A federated testbed for future networks (and 5G). https://wWW . softfire. eu/, 2018. acessed in: May 13rd, 2018.

[12] Network functions as-a-service over virtualized infrastructure. http://wWW . t-nova.eu/, 2018. acessed in: May 13rd, 2018.

[13] Unify cloud and carrier network. http://wWw.fp7-unify.eu/, 2018. acessed in: May 13rd, 2018.

[14] OpenSDNCore: Virtualization testbed for NFV/SDN environment. https:// WWW. opensdncore. org/, 2019. acessed in: May 25rd, 2019.

[15] RedIRIS-NOVA project. https://wWw.rediris.es/, 2019. acessed in: May 23rd, 2019. 
[16] Timo Ahonen, Abdenour Hadid, and Matti Pietikäinen. Face recognition with local binary patterns. In Computer Vision - ECCV 2004, pages 469-481. Springer Berlin Heidelberg, 2004.

[17] ETSI. Network function virtualization (vnf): Architectural framework. Technical Report ETSI GS NFV 002 V1.1.1 (2013-10), ETSI Industry Specification Group (ISG) Network Functions Virtualisation (NFV), 2013.

[18] Bo Han, Vijay Gopalakrishnan, Lusheng Ji, and Seungjoon Lee. Network function virtualization: Challenges and opportunities for innovations. IEEE Communications Magazine, 53:90-97, 2015.

[19] Raul Muñoz, Josep Mangues-Bafalluy, Nikolaos G. Bartzoudis, Ricard Vilalta, Ricardo Martínez, Ramon Casellas, Nicola Baldo, José Núñez-Martínez, Manuel Requena-Esteso, Oriol Font-Bach, Marco Miozzo, Pol Henarejos, Ana I. PérezNeira, and Miquel Payaró. End-to-end 5G services via an SDN/NFV-based multitenant network and cloud testbed. CoRR, abs/1803.07328, 2018.

[20] Borja Nogales, Victor Sanchez-Aguero, Ivan Vidal, and Francisco Valera. Adaptable and automated small uav deployments via virtualization. Sensors, 18(12): 4116, 2018.

[21] Borja Nogales, Victor Sanchez-Aguero, Ivan Vidal, Francisco Valera, and Jaime Garcia-Reinoso. A nfv system to support configurable and automated multi-uav service deployments. In Proceedings of the 4th ACM Workshop on Micro Aerial Vehicle Networks, Systems, and Applications, DroNet'18, pages 39-44, New York, NY, USA, 2018. ACM. ISBN 978-1-4503-5839-2. doi: 10.1145/3213526. 3213534. URL http://doi.acm.org/10.1145/3213526.3213534.

[22] Victor Sanchez-Aguero, Borja Nogales, Francisco Valera, and Ivan Vidal. Investigating the deployability of voip services over wireless interconnected micro aerial vehicles. Internet Technology Letters, 1(5):e40, 2018. doi: 10. 1002/itl2.40. URL https://onlinelibrary.wiley.com/doi/abs/ $10.1002 /$ it 12.40 . 
[23] P. Viola and M. Jones. Rapid object detection using a boosted cascade of simple features. In Proceedings of the 2001 IEEE Computer Society Conference on Computer Vision and Pattern Recognition. CVPR 2001, volume 1, pages I-I, 2001. 